\title{
Chondroid Lipoma
}

National Cancer Institute

\section{Source}

National Cancer Institute. Chondroid Lipoma. NCI Thesaurus. Code C6503.

A rare benign adipose tissue neoplasm characterized by nests and cord of abundant univacuolated and multivacuolated lipoblasts and mature adipocytes in a prominent myxoid to hyalinized chondroid matrix admix. It predominantly affects females. 